\title{
BMJ Open Time to inclusion in clinical guidance documents for non-oncological orphan drugs and biologics with expedited FDA designations: a retrospective survival analysis
}

\author{
Ryan Rodriguez (D) , ${ }^{1}$ Rachel Brunner, ${ }^{1}$ Samantha Spencer, ${ }^{1}$ Dima M Qato ${ }^{2,3}$
}

To cite: Rodriguez $\mathrm{R}$, Brunner R, Spencer S, et al. Time to inclusion in clinical guidance documents for nononcological orphan drugs and biologics with expedited FDA designations: a retrospective survival analysis. BMJ Open 2021;11:e057744. doi:10.1136/ bmjopen-2021-057744

- Prepublication history and additional supplemental material for this paper are available online. To view these files, please visit the journal online (http://dx.doi.org/10.1136/ bmjopen-2021-057744)

Received 16 0ctober 2021 Accepted 10 December 2021

Check for updates

(c) Author(s) (or their employer(s)) 2021. Re-use permitted under CC BY-NC. No commercial re-use. See rights and permissions. Published by BMJ.

${ }^{1}$ Department of Pharmacy Practice, University of Illinois Chicago College of Pharmacy, Chicago, Illinois, USA

${ }^{2}$ Program on Medicines and Public Health, Titus Family Department of Clinical Pharmacy, University of Southern California School of Pharmacy, Los Angeles, California, USA

${ }^{3}$ Leonard D. Schaeffer Center for Health Policy and Economics, University of Southern California, Los Angeles, California, USA

Correspondence to

Dr Ryan Rodriguez;

rwrodrig@uic.edu

\section{ABSTRACT}

Objectives Drug and biological products that treat rare, serious or life-threatening conditions can receive US Food and Drug Administration (FDA) orphan designation and expedited programme designations (accelerated approval, breakthrough therapy, fast track or priority review) meant to incentivise development. Timely recommendations from guidance documents may encourage more rapid and appropriate use and access to these medicines for serious conditions. We sought to determine time between FDA approval and inclusion in guidance documents for nononcological orphan products overall and by number and type of expedited programme designations.

Design and setting Retrospective survival analysis of non-oncological orphan products with $\geq 1$ expedited designation approved since 1992. In June 2020, PubMed, Turning Research into Practice and Guideline Central databases were searched to identify guidance documents influencing US practice that included each product.

Main outcomes and measures The primary outcome was time to guidance inclusion, defined as any recommendation on use provided within the recommendation framework used by the guidance document.

Results Among 135 included non-oncological orphan products, $97.0 \%(n=131)$ were designated with priority review, $49.6 \%(n=67)$ fast track, $16.3 \%(n=22)$ breakthrough therapy and $14.1 \%(n=19)$ accelerated approval. Sixty per cent of products $(n=81)$ received $\geq 2$ designations. Overall, $74.1 \%(n=100)$ were included in a guidance document. The median time to inclusion was 2.87 years (IQR 2.21-4.18) for the entire cohort. In survival analyses, guidance inclusion was more likely to occur earlier for products with $\geq 2$ designations ( $\mathrm{HR}, 1.84 ; 95 \% \mathrm{Cl}$ 1.21 to 2.79) and for those with fast-track designation compared with priority review (HR 1.40; $95 \% \mathrm{Cl} 1.02$ to 2.0). Of 35 products not included in a guidance document, $54.3 \%(n=19)$ were approved in 2018 or later.

Conclusions Among non-oncological orphan products with priority designations, nearly 3 years had passed between FDA approval and inclusion in any guidance document. These findings suggest that despite efforts to expedite availability, appropriate access to these treatments may be delayed because of the lack of timely guidance on their use in clinical practice.

\section{Strengths and limitations of this study}

- This study evaluated Food and Drug Administrationapproved non-oncological drug and biological products with orphan and expedited designations, representing a subset of therapies that fulfil unmet medical needs.

- This study evaluated the time between product approval and inclusion of the product in relevant clinical practice guidance documents, which were identified with a systematic literature search and author screening and review.

- The study excluded non-oncological products as well as non-orphan products, which limits generalisability of findings.

- The most recently approved products may not have been approved for periods of time that were sufficient to warrant guidance updates.

\section{INTRODUCTION}

Drugs that treat rare, serious or lifethreatening diseases can present challenges in development and access. To provide manufacturers incentives to develop such drugs when otherwise few exist, regulatory programmes were created, including the Orphan Drug Act (ODA) of 1983 and the US Food and Drug Administration (FDA) expedited programmes. ${ }^{12}$

The ODA provides financial incentives, including research tax credits, access to grants, and 7-year exclusivity, to encourage development of drugs for diseases affecting fewer than 200000 patients of a specified population or subset (eg, paediatric patients) of a population. ${ }^{134}$ Expedited programmes facilitate and expedite development and FDA review of drugs that address unmet medical needs of serious or life-threatening conditions to help ensure these therapies are quickly approved and available to patients 


\begin{tabular}{ll}
\hline Table 1 Food and Drug Administration (FDA) expedited pathways ${ }^{2}$ \\
\hline Expedited programme & Programme descriptions \\
\hline Accelerated approval & $\begin{array}{l}\text { Allows products for serious conditions that fill an unmet medical need to be approved based on a } \\
\text { surrogate or intermediate clinical endpoint }\end{array}$ \\
Breakthrough therapy & $\begin{array}{l}\text { Designed to expedite the development and review of products intended to treat a serious condition } \\
\text { which may demonstrate substantial improvement over alternative available therapy }\end{array}$ \\
Fast-track designation & $\begin{array}{l}\text { Designed to facilitate the development and expedite the review of products to treat serious } \\
\text { conditions and fill an unmet medical need }\end{array}$ \\
Priority review & $\begin{array}{l}\text { Designation can be applied for products that, if approved, would be significant improvements in the } \\
\text { safety or effectiveness of treatment, diagnosis or prevention of serious conditions, and implements } \\
\text { a goal for FDA to take action on a product application within } 6 \text { months, compared with } 10 \text { months } \\
\text { under standard review }\end{array}$ \\
\hline
\end{tabular}

once it is clear that their benefits justify their risks. ${ }^{2}$ Expedited programmes include fast track, breakthrough therapy, accelerated approval and priority review designations (table 1$)^{2}$

The ODA and expedited programmes have been widely and increasingly used. Since 1987, the number of expedited programmes granted to new drugs has increased by $2.6 \%$ annually. ${ }^{5}$ In 2019, $60 \%$ of novel product approvals received at least one of the four expedited programme designations. ${ }^{6}$ Further, orphan drugs or biologics composed $43 \%$ of all novel product approvals between 2012 and 2019. ${ }^{5}$ Between the time of the passage of the ODA in 1983 and 2017, 575 drug and biological products for rare diseases have been developed, providing novel therapeutic options for rare diseases. ${ }^{3}$ Orphan drugs also have an outsized impact on spending, with increases between $4 \%$ and $10 \%$ of total prescription drug spending between 1997 and 2017. ${ }^{7}$ In 2018, orphan drug sales account for approximately $30 \%$ of the US $\$ 170$ billion in sales generated from drugs approved between 2010 and 2019. ${ }^{8}$ While similar spending data are unavailable for products with expedited programme designations, these products are also typically costly.

While the ODA and expedited programmes encourage market availability, they do not ensure appropriate patient access. For example, Chambers et al reported in a 2019 analysis that the frequency of restrictions for orphan drugs was $30 \%$ overall, and varied from $11 \%$ to $65 \%$ across 17 of the largest 20 US private health plans. ${ }^{9}$ Importantly, this analysis found that only $16 \%$ of 302 drug-indication pairs were covered the same way by all health plans. Using the same data, Chambers et al later reported that orphan drugs with expedited approval were approximately $30 \%$ more likely than those without expedited approval to have coverage restrictions. ${ }^{10}$ Another analysis reported that approximately one-quarter of indications for drugs with orphan or expedited designations had restrictions on use. ${ }^{11}$ Challenges in covering drugs with expedited designations have also been documented; these include lack of coverage for some drugs approved with expedited designations among private payers because of high cost and, consistent with the lower evidentiary standards of some designations, a lack of evidence. ${ }^{12}$
The increasing prevalence, high cost and limited evidence for drugs approved with orphan and expedited designations present challenges in determining appropriate coverage decisions. Not surprisingly, clinical data were found to be the most important factor driving benefit design for orphan drugs in a survey of commercial and public payers. ${ }^{13}$ However, studies have documented the low quality of preapproval and postapproval studies of drugs approved with orphan or expedited designations, including infrequent use of randomization, blinding and terminal clinical endpoints. ${ }^{14-16}$ These studies did not report whether coverage decisions for orphan drugs were justified and consistent with evidence-based practice; such analyses could be facilitated by recommendations from guidance documents.

Experts have also described the disconnect between the cost of orphan drugs and their clinical benefit, threatening the sustainability of healthcare systems. ${ }^{17} 18$ For example, the costs per quality-adjusted life-year were estimated in 2015 to be US\$640000 and US\$3.6 million for ivacaftor and ivacaftor/lumacaftor, respectively, two agents approved by FDA for the treatment of patients with cystic fibrosis and specific genetic mutations. ${ }^{19}$ Globally, approximately 2600 and 25000 patients, respectively, carried mutations that made them eligible for these agents. To address this issue, innovative pricing strategies have been proposed, including some that discount early market prices of orphan drugs while the evidence base is immature, and adjust prices according to subsequent research and use. ${ }^{3} 18$ The allocation of scarce resources to high-cost orphan drugs presents a challenge of balancing the health of a population with that of a small subgroup of people with orphan diseases. ${ }^{20}$ Some have suggested using higher cost-effectiveness thresholds or weighted cost-effectiveness ratios for orphan diseases to ensure these patients retain access to care. Nonetheless, these strategies are not widely applied, and would be challenging to implement during the early stages of availability of orphan drugs when evidence is limited. ${ }^{17} 20$

Confronted with these challenges, formal evidencebased recommendations from clinical practice guidances may assist policy-makers in coverage determinations for drugs with orphan and expedited designations in ways 
that balance cost, access and utility. The Institute of Medicine (IOM) states that clinical practice guidelines provide a systematic aid to making complex medical decisions, combining scientific evidence, clinicians' experience and patient values, to improve health outcomes. ${ }^{21}$ Indeed, a review of international pricing strategies and coverage of orphan drugs and personalised medicine found that clinical practice guidelines strongly influence payer decisions. ${ }^{22}$ While individual clinicians lack the resources to perform comprehensive reviews of evidence from patient, payer and public health perspectives, guideline panels do possess these resources and provide additional clinical insight from expert leaders in practice areas specific to the guideline. ${ }^{23}$ Moreover, some guideline development processes, such as Grading of Recommendations, Assessment, Development, and Evaluation (GRADE), provide recommendations in a framework that has policy-level implications. Such recommendations could facilitate coverage decisions and promote appropriate access. Therefore, we sought to evaluate the time to inclusion in clinical practice guidance documents for drug and biological products approved with orphan and expedited designations.

\section{METHODS}

\section{Data sources}

We used publicly available data from FDA Center for Drug Evaluation and Research of New Molecular Entity Drug and New Biologic Approvals. ${ }^{24}$ This dataset includes regulatory and product information for products approved from 1985 through 2019, including dates of approval, types of approval and approved indications, that were current as of 21 February 2020 at the time of data collection.

\section{Inclusion and exclusion criteria}

Products were included that were approved via a New Drug Application or Biologic License Application, granted orphan drug designation and granted 1 or more of the following expedited programme designations: fast track, accelerated approval, breakthrough therapy and priority review. The earliest available of these programmes (priority review) was instituted in 1992; thus, we excluded products approved prior to this year. Products were included regardless of brand or generic status. Products that were addressed in guidances prior to their FDA approval were excluded from analyses, as these would not have been at risk for guidance inclusion at the time of their approval.

We considered the potential for individual products to be approved for more than 1 indication, and the possibility that information supporting the first approval may influence the decision for guideline panels to act on information from a second approval. Therefore, we planned to analyse only the first approved indication of a drug should this situation occur.
The major guidance documents for oncology indications in the USA are produced by the National Comprehensive Cancer Network (NCCN); these are not indexed in publicly searchable databases (eg, PubMed), nor are historical documents available in archived format at the NCCN website. This precludes the ability to identify historical and contemporaneous guidances that were published at the time of earlier product approvals. Therefore, we excluded products approved for oncology indications.

\section{Identification of guidance documents}

We searched for guidance documents (including full guidelines as well as focused updates and similar documents) influencing US practice that were applicable to each product's approved indication in order to determine the time to first inclusion in a guidance document. Our systematic search strategy intended to identify the most contemporaneous guidance documents following each product's approval. To achieve this, we searched PubMed, which would provide a comprehensive historical database of publications. We searched, as secondary resources, the Turning Research into Practice database and Guideline Central. These guideline repositories were prioritised after PubMed because they often remove outdated guidances, preventing our ability to identify contemporaneous documents.

Our PubMed search strategy involved appending a customised search string with terms for each product indication to a standardised search string including a filter for identifying guidance documents (online supplemental file 1) and a filter for a time period applicable to each product approval. This time period filter included a 6-month lookback period prior to the product's approval (to capture any guidances made in anticipation of a product's impending approval) and continued until 30 June 2020. The customised search string for each product indication was created and reviewed by at least two authors. Candidate publications were collected and reviewed.

\section{Outcome measures}

The outcome of interest was the time to first clinical recommendation for a product in guidance documents from organisations influencing US practice, which we considered as any recommendation for or against clinical use or a statement that evidence is insufficient to make a recommendation as performed within the recommendation framework used by the guidance document (eg, GRADE). When available, we recorded this information and calculated the time between product approval and publication date for the first inclusion in a guidance document. Products with no applicable guidance document or no inclusion in a guidance document by the time of our search were considered censored.

\section{Analysis}

We calculated median times to guidance document inclusion overall and for each separate expedited programme designation. Kaplan-Meier survival curves were prepared 
Table 2 Characteristics of non-oncology orphan drugs and biologics approved with expedited designations

\begin{tabular}{lc}
\hline Product characteristics & All products $(\mathbf{n = 1 3 6})$ \\
\hline Application type, $\mathrm{n}(\%)$ & $95(69.9)$ \\
NDA & $41(30.1)$ \\
BLA & \\
Expedited pathway, $\mathrm{n}(\%)$ & $131(96.3)$ \\
$\quad$ Priority review & $19(14.0)$ \\
Accelerated approval & $22(16.2)$ \\
Breakthrough therapy & $68(50.0)$ \\
Fast track & \\
\hline Total designations granted, $\mathrm{n}(\%)$ & $55(40.4)$ \\
1 & $59(43.4)$ \\
2 & $21(15.4)$ \\
3 & $1(0.7)$ \\
\hline
\end{tabular}

BLA, Biologics License Application; NDA, New Drug Application.

and compared using the log-rank test. Descriptive statistics were calculated using proportions and mean (SD) or median (IQR), as appropriate.

We performed Cox proportional hazards modelling to compute HRs for the time to guidance document inclusion for products granted 2 or more expedited programme designations versus only 1 , and for products approved with fast track, accelerated approval and breakthrough therapy designations (which permit a lower evidentiary standard) versus priority review designation (which does not modify the evidentiary standard).

\section{Patient and public involvement}

No patients were involved in the development of the research question or design of the study.

\section{RESULTS}

The FDA approved 917 new drug or biological products from 1992 through December 2019, of which 284 (30.9\%) were approved for at least 1 orphan disease. ${ }^{24}$ Eight products were addressed in a guideline before approval and were excluded from analyses. Overall, 136 non-oncology products received orphan designation and were included in the analysis. Of these, $96.3 \%(\mathrm{n}=131)$ were designated with priority review, $50.0 \% \quad(\mathrm{n}=68)$ fast track, $16.2 \%$ $(\mathrm{n}=22)$ breakthrough therapy and $14.0 \%(\mathrm{n}=19)$ accelerated approval (table 2). Two or more expedited designations were received by $59.5 \%(\mathrm{n}=81)$ of products.

Overall, $74.3 \%$ of products $(n=101)$ were addressed in a guidance document (table 3 ). The median time to guidance inclusion was 2.84 years (IQR 2.18-4.18) for the entire cohort, and did not significantly differ overall across expedited programme designations (log-rank $\mathrm{p}=0.21$; figure 1 ). However, compared with products with 1 programme designation, guidance inclusion was more
Table 3 Inclusion in clinical guidance documents for non-oncology orphan drugs and biologics approved with expedited designations

\begin{tabular}{|c|c|c|}
\hline \multicolumn{3}{|c|}{ Addressed in guidance document } \\
\hline \multicolumn{2}{|c|}{ Overall cohort, n (\%) } & $101(74.3)$ \\
\hline \multicolumn{2}{|l|}{ Priority review } & 96 (73.3) \\
\hline \multicolumn{2}{|c|}{ Accelerated approval } & $15(78.9)$ \\
\hline \multicolumn{2}{|c|}{ Breakthrough therapy } & $11(50.0)$ \\
\hline \multicolumn{2}{|l|}{ Fast track } & $51(75.0)$ \\
\hline & $\begin{array}{l}\text { Years to guidance } \\
\text { inclusion, median (IQR) }\end{array}$ & HR $(95 \% \mathrm{Cl})$ \\
\hline Overall cohort & $2.84(2.18,4.18)$ & - \\
\hline $\begin{array}{l}\text { Accelerated } \\
\text { approval }\end{array}$ & $2.18(1.68,13.49)$ & 1.3 (0.75 to 2.2$)$ \\
\hline $\begin{array}{l}\text { Breakthrough } \\
\text { therapy }\end{array}$ & $2.55(1.58, \mathrm{NE})$ & 1.3 (0.71 to 2.5$)$ \\
\hline Fast track & $2.18(1.68,3.74)$ & 1.4 (1.02 to 2.0$)$ \\
\hline Priority review & $2.87(2.55,4.71)$ & Ref \\
\hline \multicolumn{3}{|l|}{$\begin{array}{l}\text { Number of } \\
\text { designations }\end{array}$} \\
\hline$\geq 2$ & $2.21(1.97,2.88)$ & 1.78 (1.18 to 2.69$)$ \\
\hline 1 & $4.18(3.44,7.79)$ & Ref \\
\hline
\end{tabular}

NE, not estimable.

likely to occur earlier for products with $\geq 2$ designations compared with those with 1 designation (median, 2.21 vs 4.18 years, respectively; Cox proportional HR, 1.78; 95\% CI 1.18 to 2.69; figure 2). Compared with products approved with priority review, only those with fast-track

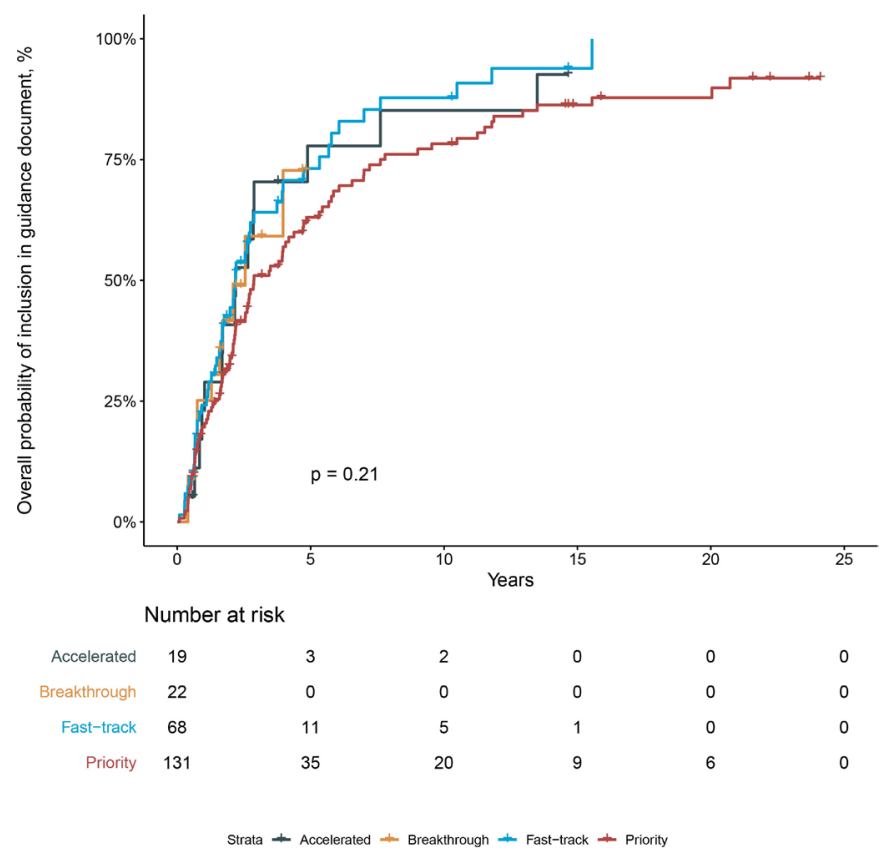

Figure 1 Kaplan-Meier plot of inclusion in guidance documents for orphan products approved via expedited pathways. 


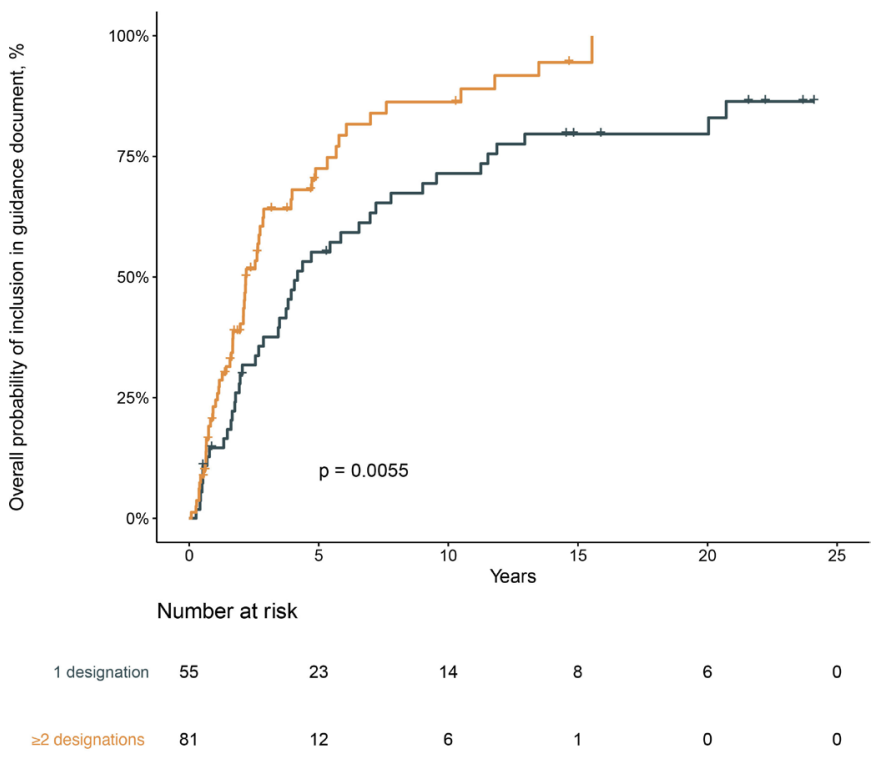

Strata +1 designation -22 designation

Figure 2 Kaplan-Meier plot of inclusion in guidance documents for orphan products approved with 1 or $\geq 2$ expedited designations.

designation were more likely to be included earlier in guidance documents (HR, 1.40; 95\% CI 1.02 to 2.0).

Of 35 products not included in a guidance document, $54.3 \%(\mathrm{n}=19)$ were approved in 2018 or later (table 4); other characteristics were similar to those of products included in a guidance document. A majority of these products received either priority review designation alone $(\mathrm{n}=35)$ or both priority review and fast-track designations $(\mathrm{n}=32)$.

\section{DISCUSSION}

To our knowledge, this is the first study to describe time between approval of orphan products with expedited designations and their inclusion in clinical practice guidance documents. These findings have importance in the current regulatory environment. The annual number of orphan drug designations assigned by FDA has increased from roughly 60 in 2002 to 427 in 2017. ${ }^{1}$ Additionally, the mean annual growth rate of orphan products has been predicted to increase by $11.1 \%$ between 2017 and 2022, significantly higher than that predicted for non-orphan products $(5.3 \%)$.

The roughly 3-year period for guidance inclusion could be due, in part, to the resource investment demanded by rigorous guideline development. ${ }^{21}$ The mobilisation of key stakeholders and contributors can be a lengthy process. However, it can be streamlined; for example, NCCN guidelines provide in-depth, transparent methods for guideline development and committee mobilisation. ${ }^{25}$ The NCCN guidance documents are updated at least annually and often in response to new product approvals. This facilitates timely decision-making and can serve as
Table 4 Characteristics of non-oncology orphan drugs and biologics not included in guidance documents

\begin{tabular}{lc}
\hline Product characteristics & All products (n=136) \\
\hline Total NDA and BLA approvals, $n$ (\%) & 35 \\
\hline NDA & $27(77.1)$ \\
\hline BLA & $8(22.9)$ \\
\hline Expedited pathway, $n$ (\%) & \\
\hline Priority review & $35(100.0)$ \\
\hline Accelerated approval & $4(11.4)$ \\
\hline Breakthrough therapy & $11(31.4)$ \\
\hline Fast track & $17(48.6)$ \\
\hline Total designations granted, $n$ (\%) & \\
\hline 1 & $12(34.3)$ \\
\hline 2 & $15(42.9)$ \\
\hline 3 & $7(20.0)$ \\
\hline 4 & $1(2.9)$ \\
\hline Approval year, $n$ (\%) & \\
\hline 1996 & $2(5.7)$ \\
\hline 1998 & $2(5.7)$ \\
\hline 2004 & $2(5.7)$ \\
\hline 2005 & $3(8.6)$ \\
\hline 2010 & $1(2.9)$ \\
\hline 2015 & $3(8.6)$ \\
\hline 2016 & $1(2.9)$ \\
\hline 2017 & $2(5.7)$ \\
\hline 2018 & $8(22.9)$ \\
\hline 2019 & $11(31.4)$ \\
\hline Years since approval, median (IQR) & $2.20(1.13,12.42)$ \\
\hline
\end{tabular}

BLA, Biologics License Application; NDA, New Drug Application.

a model for other guideline panels. Other explanations for the delay between approval and guidance provision may relate to the limited evidence available at the time of product approval. Recommendations for use of orphan drugs should be supported by robust evidence; such evidence may not be immediately available, and professional societies may deem full guidance production or revision unjustified. Nonetheless, statements regarding the limited evidence and recommendations for restricted, yet appropriate, use can still be useful to policy-makers in justifying coverage decisions.

We also found that orphan products with 2 or more expedited designations were included more quickly into guidance documents compared with those with fewer designations. This may suggest that societies recognise an additive effect of multiple designations. However, we found limited differences between expedited designations; therefore, it is unclear how the quality rather than quantity of expedited designations may influence the time to guidance document inclusion. 
The IOM provides recommendations on updating guidelines in Clinical Practice Guidelines We Can Trust. ${ }^{21}$ The IOM states that literature should be monitored regularly to identify new and relevant evidence, and guidance should be updated when evidence suggests need for modification of a clinically important recommendation. Guidance revision may be needed when a recommended intervention causes previously unknown harm, and when a recommendation applies to new populations. The first of these situations is reflected in findings by Mostaghim et $a l$ which identified a $38 \%$ increased rate of safety-related label changes for products approved via expedited pathways. ${ }^{26}$ The second is represented by the fact that orphan products are by nature the first treatments available for a population. To optimise market entry of new medications, Godman et al in a review of access barriers to new medicines, suggested postmarketing evaluation of prescribing practices against current guidelines. ${ }^{27}$ Thus, approval of products such as those in our analysis could prompt initiation or revision of guidance documents.

The availability of clinical recommendations from professional societies could be considered along with other factors in broader policy and formulary deliberations related to orphan drugs. For example, multicriteria decision analysis (MCDA) is an explicit framework used to evaluate the utility of health technologies to determine whether they merit funding. ${ }^{1728}$ Approaches using MCDA incorporate weighted scores for various criteria to generate a composite score that facilitates comparison of health technologies. Criteria considered in MCDA may include clinical benefit and safety, quality of evidence, implementation feasibility, innovation, clinical need and societal and ethical values. ${ }^{28}$ The availability of recommendations from respected professional societies might play a supporting role in MCDA.

Several products in this analysis serve as useful examples of rapid and delayed inclusion in guidance documents. For example, pirfenidone, a treatment for idiopathic pulmonary fibrosis that received priority review, breakthrough therapy, and fast-track designations, was included in an international guideline 8 months after its approval. ${ }^{29}$ The publication of new evidence was a predefined surveillance criterion for updates according to the previous version of the guideline.

In contrast, eteplirsen, approved for the treatment of Duchenne muscular dystrophy (DMD) in patients with a confirmed mutation amenable to exon 51 skipping, has yet to be addressed in guidance documents. ${ }^{24}$ Eteplirsen received priority review, accelerated approval, and fasttrack designations, and was approved in September 2016 based on a trial including 12 children that evaluated the surrogate outcome of increased dystrophin in skeletal muscle. ${ }^{30-32}$ Many insurers declined coverage or imposed restrictions on use of eteplirsen due, at least in part, to this limited evidence. ${ }^{30} 33$ Advocacy groups and parents disputed coverage decisions because of its high cost. ${ }^{33}$ While various factors contributed to the uncertain value of eteplirsen, earlier guidance from professional societies may have alleviated some of these challenges. Since the approval of eteplirsen, 3 additional antisense oligonucleotides have been approved to treat DMD. ${ }^{34-36}$ To date, these therapies have not been addressed in guidance documents.

There are challenges to providing recommendations for use of products in this analysis. Orphan diseases are less likely to be represented by a professional organisation equipped to develop a clinical practice guideline. ${ }^{21}$ Additionally, orphan drug approvals are often based on clinical studies with less rigorous designs or results based on surrogate endpoints. With limited evidence presented at approval for some orphan products, the role of expert opinion in guideline development may be outsized compared with its role in non-orphan products.

Our study has several limitations. First, the development of search strings and manual screening present risk of subjectivity in identifying relevant documents. However, we attempted to mitigate this through review of each search string by at least two authors and secondary review of selected documents. Second, oncological products were excluded from our search strategy because of the inability to retrieve historic guidelines from NCCN, a primary guidance for oncology practice in the USA. This prohibits generalisability to oncology products. Additionally, we restricted our analysis to products with both orphan and expedited designations, limiting generalisability to non-orphan products. Finally, our analyses identified that the median time to guidance inclusion was almost 3 years. Thus, products approved in recent years may have not had sufficient time to be included in guidances at the time of data collection. However, specific cases, such as that of pirfenidone, suggest that a strategic approach could promote more rapid inclusion of recent approvals in guidance documents.

In conclusion, we found that for FDA-approved orphan products with expedited programme designations, approximately 3 years elapsed between product approval and inclusion in guidance documents; guidance inclusion was more likely to occur earlier for products with $\geq 2$ versus those with 1 designation, and for those with fast-track versus priority review designation. More timely development of guidance documents after approval of these products could encourage more rapid and appropriate uptake into practice and could be initiated when FDA approval is anticipated. Further research is needed to better characterise barriers to inclusion of these products in guidance documents and subsequently, their appropriate use and access.

Contributors RR contributed to the conceptualisation of the research question, was responsible for project administration, and is guarantor of the overall content. All authors contributed to development of the protocol and wrote and reviewed the manuscript, and approved the final manuscript. RB, RR and SS collected and organised data. RR performed all analyses. DMQ, RB, RR reviewed and approved results. RR accepts full responsibility for the finished work and the conduct of the study. All authors had access to the data, and controlled the decision to publish.

Funding The authors have not declared a specific grant for this research from any funding agency in the public, commercial or not-for-profit sectors. 
Competing interests RR declares spouse's employment at Melinta Therapeutics. $\mathrm{DMQ}, \mathrm{RB}$, and SS declare no competing interests.

Patient consent for publication Not applicable.

Ethics approval A local Institutional Review Board (UIC Office for the Protection of Research Subjects) determined the study did not constitute human subject research (protocol \# 2020-1168).

Provenance and peer review Not commissioned; externally peer reviewed.

Data availability statement Data are available upon reasonable request. Data are available from the corresponding author on reasonable request from researchers providing a methodologically sound proposal. Data regarding orphan and expedited designations were retrieved from the US Food and Drug Administration website. Data regarding product inclusion in guidance documents were retrieved via manual review of full-text articles identified through systematic literature searches.

Supplemental material This content has been supplied by the author(s). It has not been vetted by BMJ Publishing Group Limited (BMJ) and may not have been peer-reviewed. Any opinions or recommendations discussed are solely those of the author(s) and are not endorsed by BMJ. BMJ disclaims all liability and responsibility arising from any reliance placed on the content. Where the content includes any translated material, BMJ does not warrant the accuracy and reliability of the translations (including but not limited to local regulations, clinical guidelines, terminology, drug names and drug dosages), and is not responsible for any error and/or omissions arising from translation and adaptation or otherwise.

Open access This is an open access article distributed in accordance with the Creative Commons Attribution Non Commercial (CC BY-NC 4.0) license, which permits others to distribute, remix, adapt, build upon this work non-commercially, and license their derivative works on different terms, provided the original work is properly cited, appropriate credit is given, any changes made indicated, and the use is non-commercial. See: http://creativecommons.org/licenses/by-nc/4.0/.

\section{ORCID iD}

Ryan Rodriguez http://orcid.org/0000-0002-6333-2577

\section{REFERENCES}

1 Attwood MM, Rask-Andersen M, Schiöth HB. Orphan drugs and their impact on pharmaceutical development. Trends Pharmacol Sci 2018;39:525-35

2 US Food and Drug Administration. Guidance for industry: expedited programs for serious conditions - drugs and biologics, 2014. Available: https://www.fda.gov/media/86377/download [Accessed 18 Aug 2021].

3 Luzzatto L, Hyry HI, Schieppati A, et al. Outrageous prices of orphan drugs: a call for collaboration. Lancet 2018;392:791-4.

4 US Food and Drug Administration. Guidance for industry: clarification of orphan designation of drugs and biologics for pediatric subpopulations of common diseases, 2018. Available: https://www. fda.gov/files/about\%20fda/published/Clarification-of-OrphanDesignation-of-Drugs-and-Biologics-for-Pediatric-Subpopulationsof-Common-Diseases.pdf [Accessed 15 Nov 2021]

5 Kesselheim AS, Wang B, Franklin JM, et al. Trends in utilization of FDA expedited drug development and approval programs, 19872014: cohort study. BMJ 2015;351:h4633.

6 US Food and Drug Administration. New drug therapy approvals 2019, 2020. https://www.fda.gov/drugs/new-drugs-fda-cders-newmolecular-entities-and-new-therapeutic-biological-products/newdrug-therapy-approvals-2019\#2019

7 Chambers JD, Silver MC, Berklein FC, et al. Orphan drugs offer larger health gains but less favorable cost-effectiveness than nonorphan drugs. J Gen Intern Med 2020;35:2629-36.

8 Brown DG, Wobst HJ. A decade of FDA-approved drugs (20102019): trends and future directions. J Med Chem 2021;64:2312-38.

9 Chambers JD, Panzer AD, Kim DD, et al. Variation in US private health plans' coverage of orphan drugs. Am J Manag Care 2019;25:508-12.

10 Chambers JD, Margaretos NM, Enright DE. Is an orphan drug's cost-effectiveness associated with us health plan coverage Restrictiveness? Pharmacoeconomics 2021 doi:10.1007/s40273021-01096-5

11 Chambers JD, Kim DD, Pope EF, et al. Specialty drug coverage varies across commercial health plans in the US. Health Aff 2018;37:1041-7

12 Gellad WF, Kesselheim AS. Accelerated approval and expensive drugs - a challenging combination. N Engl J Med 2017;376:2001-4.
13 Hyde R, Dobrovolny D. Orphan drug pricing and payer management in the United States: are we approaching the tipping point? Am Health Drug Benefits 2010;3:15-23.

14 Dupont AG, Van Wilder PB. Access to orphan drugs despite poor quality of clinical evidence. Br J Clin Pharmacol 2011;71:488-96.

15 Naci H, Wouters OJ, Gupta R, et al. Timing and characteristics of cumulative evidence available on novel therapeutic agents receiving food and drug administration accelerated approval. Milbank $Q$ 2017;95:261-90.

16 Pontes C, Fontanet JM, Vives R, et al. Evidence supporting regulatory-decision making on orphan medicinal products authorisation in Europe: methodological uncertainties. Orphanet J Rare Dis 2018;13:206.

17 Godman B, Hill A, Simoens S, et al. Potential approaches for the pricing of cancer medicines across Europe to enhance the sustainability of healthcare systems and the implications. Expert Rev Pharmacoecon Outcomes Res 2021;21:527-40.

18 EURORDIS Rare Diseases Europe. Breaking the access deadlock to leave no one behind. EURORDIS rare diseases Europe, 2018. Available: https://www.eurordis.org/accesspaper\#: :text=Breaking\% 20the\%20Access\%20Deadlock\%20to\%20Leave\%20No\%20One\% 20Behind\&text=In\%20the\%20paper\%2C\%20EURORDIS\%20calls and\%20the\%20European\%20Medicines\%20Agency [Accessed 14 Nov 2021].

19 Hollis A. Orphan drug pricing and costs: a case study of Kalydeco and Orkambi. Healthc Policy 2019;15:70-80.

20 Simoens S, Picavet E, Dooms M, et al. Cost-effectiveness assessment of orphan drugs: a scientific and political conundrum. Appl Health Econ Health Policy 2013;11:1-3.

21 Institute of Medicine Committee on Standards for Developing Trustworthy Clinical Practice Guidelines. Current best practices and standards for development of trustworthy CPGs: part II, traversing the process. In: Graham R, Mancher M, Miller Wolman D, et al, eds. Clinical practice guidelines we can trust. National Academies Press (US), 2011. https://www.nap.edu/catalog/13058/clinical-practiceguidelines-we-can-trust

22 Degtiar I. A review of international coverage and pricing strategies for personalized medicine and orphan drugs. Health Policy 2017;121:1240-8.

23 GRADE Working Group. GRADE handbook for grading quality of evidence and strength of recommendations, 2013. Available: https:// gdt.gradepro.org/app/handbook/handbook.html

24 US Food and Drug Administration. Compilation of CDER new molecular entity (NME) drug and new biologic approvals, 2020 Available: https://www.fda.gov/drugs/drug-approvals-anddatabases/compilation-cder-new-molecular-entity-nme-drug-andnew-biologic-approvals [Accessed 18 Aug 2021].

25 National Comprehensive Cancer Network. About clinical practice guidelines. Available: https://www.nccn.org/guidelines/guidelinesprocess/about-nccn-clinical-practice-guidelines [Accessed 18 Aug 2021].

26 Mostaghim SR, Gagne JJ, Kesselheim AS. Safety related label changes for new drugs after approval in the US through expedited regulatory pathways: retrospective cohort study. BMJ 2017;358:j3837.

27 Godman B, Bucsics A, Vella Bonanno P, et al. Barriers for access to new medicines: searching for the balance between rising costs and limited budgets. Front Public Health 2018;6:328.

28 Howard S, Scott IA, Ju H, et al. Multicriteria decision analysis (MCDA) for health technology assessment: the Queensland health experience. Aust Health Rev 2019;43:591-9.

29 Raghu G, Rochwerg B, Zhang Y, et al. An official ATS/ERS/JRS/ALAT clinical practice guideline: treatment of idiopathic pulmonary fibrosis. An update of the 2011 clinical practice guideline. Am J Respir Crit Care Med 2015;192:e3-19.

30 Dowling JJ. Eteplirsen therapy for Duchenne muscular dystrophy: skipping to the front of the line. Nat Rev Neurol 2016;12:675-6.

31 Mendell JR, Rodino-Klapac LR, Sahenk Z, et al. Eteplirsen for the treatment of Duchenne muscular dystrophy. Ann Neurol 2013;74:637-47

32 U.S. Food \& Drug Administration. FDA grants accelerated approval to first drug for Duchenne muscular dystrophy, 2016. Available: https://www.fda.gov/news-events/press-announcements/fda-grantsaccelerated-approval-first-drug-duchenne-muscular-dystrophy [Accessed 18 Aug 2021].

33 Thomas K. Insurers battle families over costly drug for fatal disease, 2017. https://www.nytimes.com/2017/06/22/health/duchennemuscular-dystrophy-drug-exondys-51.html

34 Vyondys 53. Package insert. Sarepta Therapeutics Inc, 2021.

35 Amondys 45. Package insert. Sarepta Therapeutics Inc, 2021.

36 Viltepso. Package insert. NS Pharma Inc, 2021. 